\title{
Case Report \\ Favourable Experience with M-Mode Sonography in the Diagnosis of Pneumothorax in Two Patients with Thoracic Subcutaneous Emphysema
}

\author{
T. Berlet and R. Etter \\ Department of Intensive Care Medicine, Inselspital, Bern University Hospital and University of Bern, 3010 Bern, Switzerland \\ Correspondence should be addressed to T. Berlet; thomas.berlet@insel.ch
}

Received 20 August 2014; Accepted 30 October 2014; Published 13 November 2014

Academic Editor: Roberto Grassi

Copyright (C) 2014 T. Berlet and R. Etter. This is an open access article distributed under the Creative Commons Attribution License, which permits unrestricted use, distribution, and reproduction in any medium, provided the original work is properly cited.

\begin{abstract}
Introduction. Thoracic subcutaneous emphysema may be caused by various pathologies. In mechanically ventilated patients, timely diagnostic workup is particularly important, as the presence of subcutaneous surgical emphysema may indicate pneumothorax, representing a risk factor for the development of life threatening tension pneumothorax. Thoracic ultrasound is of proven value for the detection of pneumothorax but has so far been considered of little value in the context of subcutaeneous emphysema, due to poor visibility of anatomic structures. Case Presentation. We present the successful use of diagnostic M-mode sonography in two mechanically ventilated patients who developed thoracic subcutaneous emphysema. In both cases B-mode sonography was inconclusive. Conclusion. M-mode sonography may be more sensitive than B-mode sonography in the detection of lung sliding and could become a useful diagnostic tool when pneumothorax needs to be ruled in or ruled out and visibility of the pleura is limited.
\end{abstract}

\section{Introduction}

Thoracic subcutaneous emphysema may be caused by injuries to the airways, pharynx, oesophagus, abdomen or retroperitoneal space, severe soft-tissue infections, or pneumothorax [1]. In mechanically ventilated patients, the presence of subcutaneous surgical emphysema is always a cause for concern as it may indicate pneumothorax along with the risk for the development of life threatening tension pneumothorax. Physical examination and portable plain chest X-ray are both frequently unreliable in this scenario. CT-scanning, while highly sensitive and specific, is usually not feasible in this situation [2]. Thoracic ultrasound is of proven value for the diagnosis of pneumothorax [3]. However caution is warranted in the setting of thoracic subcutaneous emphysema due to poor visibility of anatomic structures and particularly of the pleura [4]. We report our favourable experience with the use of M-mode sonography to rule in and rule out pneumothorax in two mechanically ventilated patients who developed subcutaneous emphysema. In both cases a portable Sonosite M-Turbo ultrasound unit (SonoSite Inc.,
Bothell, WA, USA) in connection with L-38 broadband linear array $5-10 \mathrm{MHz}$ transducer was used.

\section{Case Reports}

Case 1. A 54-year-old male was admitted to our ICU following emergency coronary bypass artery grafting for a myocardial infarct complicated by acute heart failure. He remained on mechanical ventilation for 8 days. Following reinsertion of a central venous catheter into the right subclavian vein on day 6, subcutaneous emphysema developed; it was located predominantly at the front of the patient's left hemithorax. Thoracic ultrasonography was performed to rule out pneumothorax as a possible cause. In B-mode sonography, the pleural lines were barely visible bilaterally, despite the operator's effort to displace the subcutaneous gas by applying pressure to the soft tissue with the transducer. Lung sliding could not be identified with confidence. Neither comet-tail artifacts nor lung point nor lung pulse could be seen (see additional 


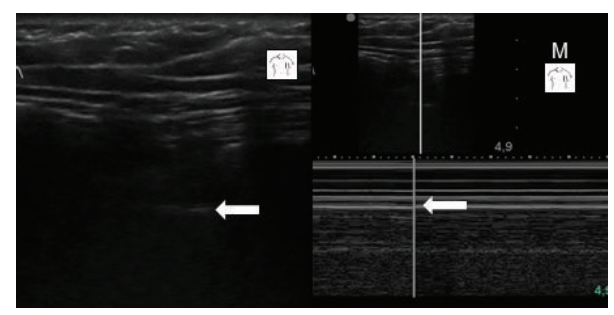

FIgURE 1: Case 1: B-mode and M-mode sonography using a linear transducer positioned in the mid-clavicular line at the $3 \mathrm{rd} \mathrm{left}$ intercostal space. Arrows indicate the pleural lines (B: B-mode, M: M-mode).

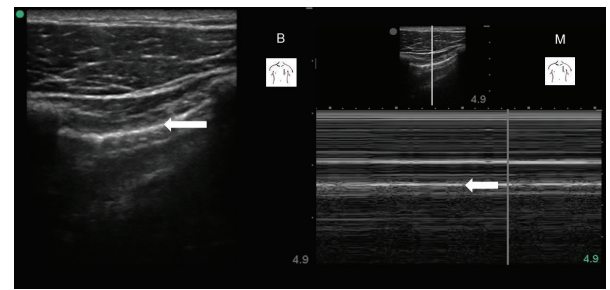

Figure 2: Healthy volunteer for comparison: B-mode and Mmode sonography using a linear transducer positioned in the midclavicular line at the $3 \mathrm{rd}$ intercostal space. Arrows indicate the pleural lines (B: B-mode, M: M-mode).

electronic content "Case 1" in Supplementary Material available online at http://dx.doi.org/10.1155/2014/906127). In Mmode sonography, however, signs of lung-sliding ("sea-shore sign") were easily visualised. In fact, the ultrasound pattern in M-mode sonography was almost as distinct as in a healthy volunteer (Figures 1 and 2). Emphysema subsided in the subsequent days without the need for an intervention.

Case 2. A 73-year-old male who underwent chemotherapy for chronic lymphocytic leukaemia was admitted to our ICU with hypoxaemic respiratory failure caused by hospital acquired pneumonia. Invasive mechanical ventilation had to be initiated on day two. Acute respiratory distress syndrome (ARDS) ensued. On day 10 the patient developed right sided thoracic subcutaneous emphysema, accompanied by signs and symptoms of worsening respiratory function. Thoracic ultrasonography was performed to rule in or rule out pneumothorax as a possible cause. Similar to Case 1, in B-mode scanning chest wall structures and pleural lines were barely visible; the presence or absence of lung sliding could not be confirmed (see additional electronic content "Case 2"). In M-mode sonography the absence of lung sliding ("barcode signs") was detected (Figure 3). Pneumothorax was confirmed during chest tube insertion. Following the intervention the patient's respiratory condition improved rapidly.

\section{Discussion}

Our experience confirms the limited ability of B-mode sonography to confirm or exclude pneumothorax when

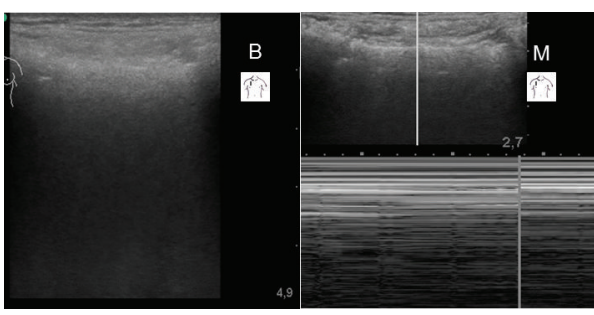

Figure 3: Case 2: B-mode and M-mode sonography using a linear transducer positioned in the mid-clavicular line at the $3 \mathrm{rd}$ right intercostal space (B: B-mode, M: M-mode).

thoracic subcutaneous emphysema is present [4]. While both M-mode and Colour Doppler sonography have been described as alternative modes in the examination of the pleura, no suggestion has been made so far that these modes might carry superior diagnostic capabilities in terms of sensitivity or specificity [5-7]. When we applied M-mode sonography after localising the intercostal spaces with Bmode sonography we were able to correctly diagnose the absence and strongly suspect the presence of pneumothorax in two very challenging clinical situations without difficulties. Our explanation for this phenomenon is twofold.

Firstly, the single ultrasound beam of M-mode sonography allows the operator to focus on a suitable acoustic window, whereas the large acoustic footprint of B-mode sonography causes the field of view to be "crowded" by reverberation artifacts originating from subcutaneous gas bubbles. Secondly, the high temporal resolution of M-mode sonography as compared to $2 \mathrm{D}$ techniques facilitates the recognition of even subtle motion artifacts caused by lung sliding [8]. A possible disadvantage of M-mode sonography could be the difficulty to accurately localise the pleural interface. This would be mainly due to the presence of multiple hyperechoic lines along the path of the ultrasound beam, each representing an interface between two tissue layers with different acoustic properties.

\section{Conclusion}

We conclude that M-mode sonography is more sensitive than B-mode sonography in detecting even subtle motion artifacts caused by lung sliding, may be a useful diagnostic tool for pneumothorax when visibility of the pleura is limited, and merits systematic evaluation regarding its overall diagnostic accuracy.

\section{Conflict of Interests}

The authors declare that they have no conflict of interests.

\section{References}

[1] M. H. Baumann and M. Noppen, "Pneumothorax," Respirology, vol. 9, no. 2, pp. 157-164, 2004.

[2] G. Volpicelli, "Sonographic diagnosis of pneumothorax," Intensive Care Medicine, vol. 37, no. 2, pp. 224-232, 2011. 
[3] G. Volpicelli, M. Elbarbary, M. Blaivas et al., "International evidence-based recommendations for point-of-care lung ultrasound," Intensive Care Medicine, vol. 38, no. 4, pp. 577-591, 2012.

[4] D. A. Lichtenstein, G. Mezière, N. Lascols et al., "Ultrasound diagnosis of occult pneumothorax," Critical Care Medicine, vol. 33, no. 6, pp. 1231-1238, 2005.

[5] M. Lyon, S. A. Shiver, and P. Walton, "M-mode ultrasound for the detection of pneumothorax during helicopter transport," The American Journal of Emergency Medicine, vol. 30, no. 8, pp. 1577-1580, 2012.

[6] J. Cunningham, A. W. Kirkpatrick, S. Nicolaou et al., "Enhanced recognition of "lung sliding" with power color Doppler imaging in the diagnosis of pneumothorax," Journal of Trauma: Injury Infection \& Critical Care, vol. 52, no. 4, pp. 769-771, 2002.

[7] M. Kreuter, R. Eberhardt, H. Wenz, H. Schmitteckert, C.P. Heussel, and F. Herth, "Diagnostic value of transthoracic ultrasound compared to chest radiography in the detection of a post-interventional pneumothorax," Ultraschall in der Medizin, vol. 32, supplement 2, pp. E20-E23, 2011.

[8] O. Rider, "Ultrasound," in Echocardiography, P. Leeson, D. Augustine, A. R. J. Mitchell, and H. Becher, Eds., Oxford University Press, Oxford, UK, 2012. 


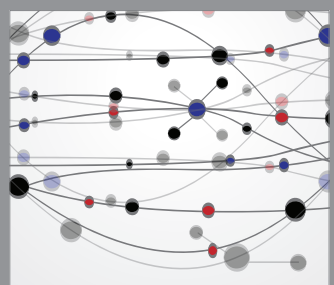

The Scientific World Journal
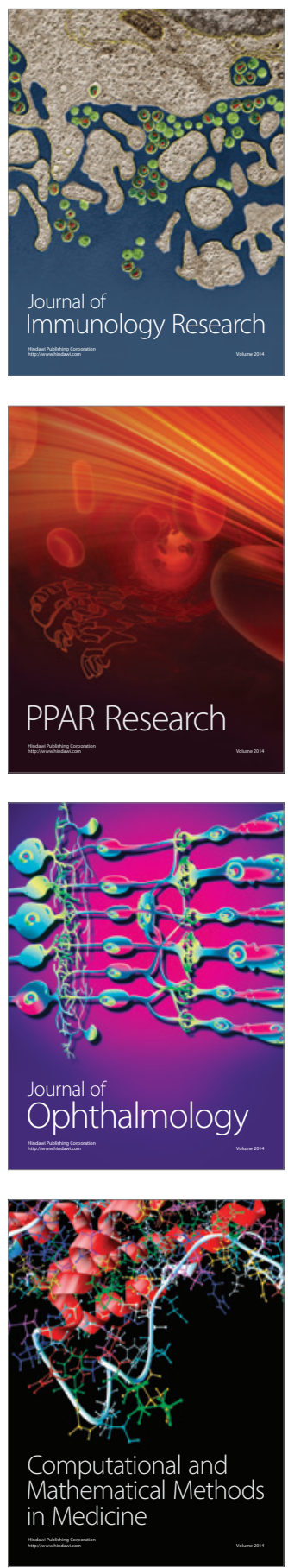

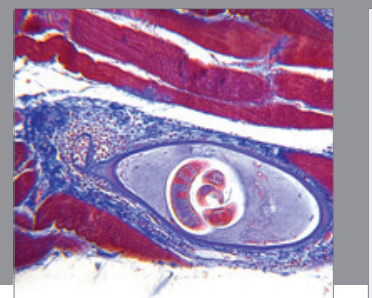

Gastroenterology

Research and Practice
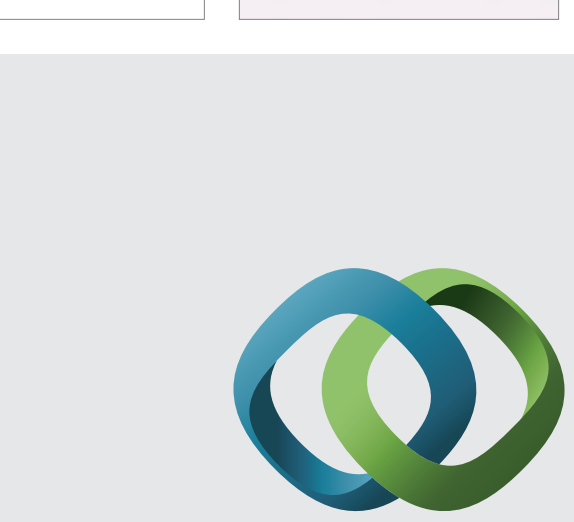

\section{Hindawi}

Submit your manuscripts at

http://www.hindawi.com
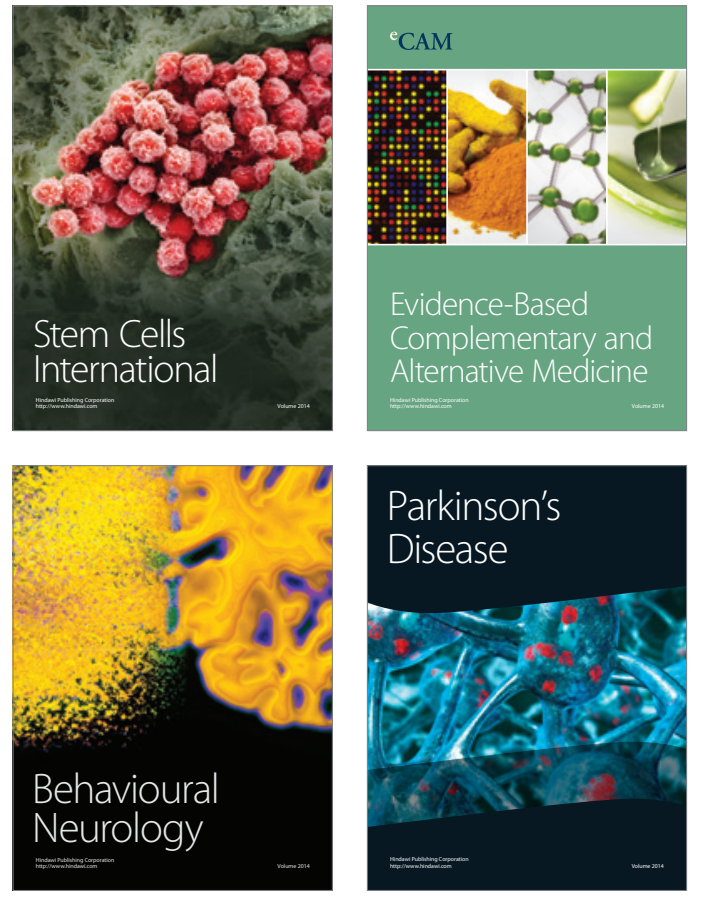
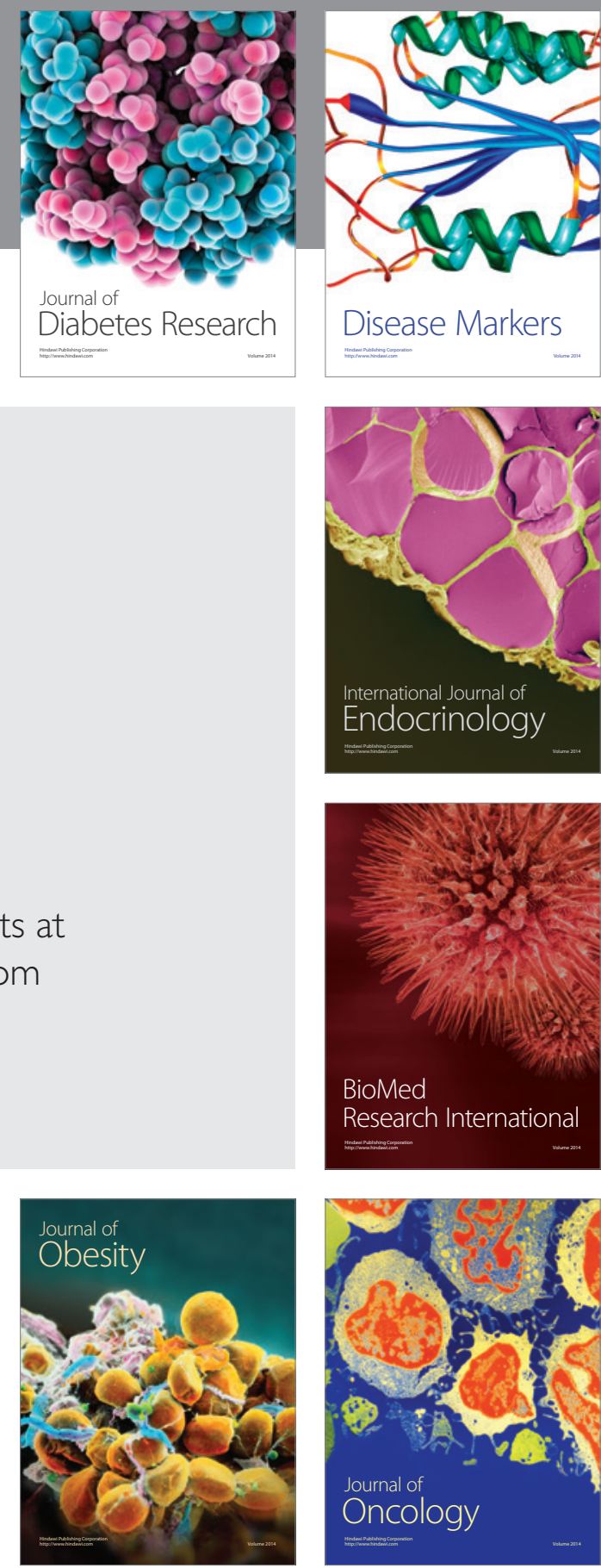

Disease Markers
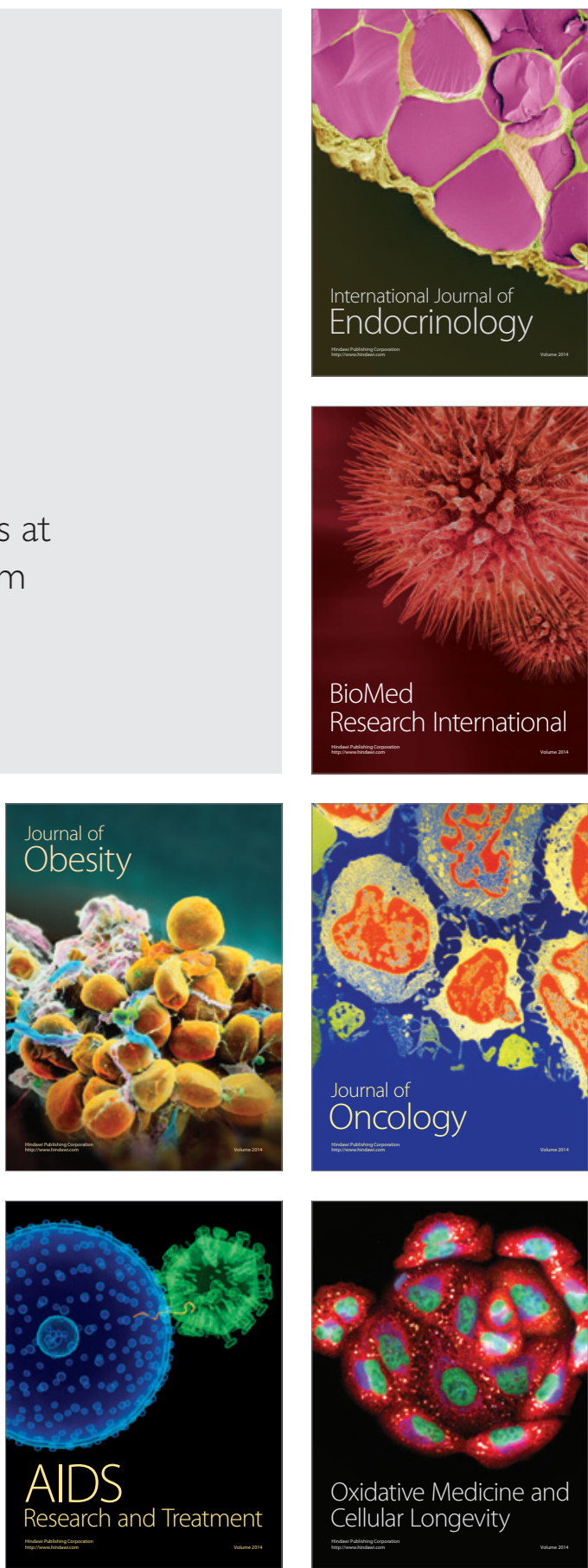